4 times due to line infection only without neutropenia. Most infected central line lumen is red lumen 52\%, white lumen $36 \%$ and blue lumen $12 \%$ respectively. Most common organism causing infection is Gram positive cocci in both Red \& White lumen whereas most common infection in blue lumen is caused by Gram negative bacilli. Gram positive cocci the most common micro organism causing infection is staphylococcus. Gram negative bacilli most common micro organism causing infection are E.coli \& Elizabethkingia.

Conclusion Covid 19 PCR was tested in all admitted oncology patients, out of 20 total patients in Wexford area 9 patients were admitted and all were tested negative for Covid 19 PCR. There is no relation of Covid 19 infection on oncology patients that are admitted in Wexford General hospital. There is no relation of Covid 19 infection on newly diagnosed oncology patients, as on time of their diagnosis they were all tested negative for Covid 19 PCR.

Main cause of admission is $71 \%$ line infection out of which $52 \%$ is related to febrile neutropenia and $28 \%$ are newly diagnose oncology patients. Highest number of patients admitted have diagnosis of Acute lymphoblastic Leukemia (Newly diagnosed as well as previously diagnosed ALL with line infection and febrile neutropenia). Number of average percentage of inpatient admission in Wexford General Hospital from January 2020 till January 2021 are as follows: 63\%. ALL, 32\% Medullobastoma,2\% Astrocytoma. 6 out of 9 are newly diagnosed cases and 3 are previously diagnosed oncology patients admitted due to infection ( line infection with or without febrile neutropenia).

Overall male predominance of oncology patients i.e 12 out of 20 patients are male. Medulloblastoma predominance in male, no female patient diagnose with it. Acute Lymphoblastic Leukemia has male predominance i.e 3 out of 4 male and only 1 female out of 4 ALL patients. Hodgkin and Non Hodgkin Lymphoma only male predominance.

Wilms tumor, neuroblastoma, AML only female predominance, no male patients are diagnosed with it.

Blood transfusion was given only to ALL patients, $46 \%$ pack RBC was transfused and 54\% platelet transfusion was given to patient admitted in Wexford General Hospital in Paediatric ward. There is no relation of anemia and thrombocytopenia in oncology patients as they were all tested negative for Covid 19 PCR test. Total number of bed days occupied from Janruary 2020 to Janruary 2021 is 93 days out which ALL patients has occupied highest number of bed days i.e $60 \%$ whereas Astrocytoma has occupied least number of bed days i.e $2 \%$.

Recommendation In view of novel Covid 19 infection and its multiple strains causing Pandemic this audit should be repeated on yearly basis to review any impact of Covid 19 on oncology patients. To rule out any co relation of Covid 19 with newly diagnosed oncology patients. To rule out any relation of Covid 19 causing haemolysis, anemia and thrombocytopenia in oncology patients. National plan of care, supportive care to patients. Safeguarding with proper protocol providing PPE Equipment in relation to patient and staff safety. More isolation rooms availability for oncology patients as they are prone to infections and Covid 19 could be the sinister for their mortality as they are already immunocompromised. Reinforce the medical staff to decrease the workload. Psychological and social support for Oncology patients and patient carer to cope in overwhelming situation due to Covid 19 Pandemic.

\section{SEROPREVALENCE OF CYTOMEGALOVIRUS AMONG SCHOOL-AGED CHILDREN IN RUSSIAN FEDERATION}

Ekaterina Dubonosova*, L Namazova-Baranova, E Vishneva, A Pankova. Research Institute of Pediatrics and Child Health Central Clinical Hospital of the Russian Academy of Sciences, Ministry of Science and Higher Education; Pirogov Russian National Research Medical University

\subsection{6/archdischild-2021-europaediatrics.61}

Cytomegalovirus (CMV) is an important human pathogen in case of immature or compromised immune system, such as the unborn child have. We thus aimed to examine risk factors for CMV infection in young people in Russia and, in order to improve our understanding of CMV epidemiology and guide future disorder prophylaxis strategies.

Objectives To explore cytomegalovirus (CMV) seroprevalence among school-aged children in different age groups.

We conducted retrospective evaluation of the seroprevalence of CMV IgG antibodies among immunocompetent school-aged children ( $\mathrm{n}=1315)$, age group from 10 to 15 years, from different regions in Russia $(n=7)$. Children were divided into 2 groups; in the first group was children under 13 years old, in the second group - over 13 years old. Comparison of two independent groups was determined using the Mann-Whitney test and the Kruskal-Wallis test. We analyzed the prevalence of CMV serotype and risk factors for infection.

We estimated a total CMV seroprevalence of 74,6\% ( $\mathrm{n}=$ 981). The median of age in the 1 st group was 10.9 (10.6; $11,3)$, the median of age in the 2nd group was $14.9(14,6$; 15,1). CMV seroprevalence was strongly associated with age, increasing from $71,8 \%$ in the first group, throughout adolescence $(77,2 \%$ in the second group) $p=0,048$. There were no statistically significant gender differences between regions.

The results are consistent with global data and require further study. These estimates of the CMV distribution will help develop national and regional models and algorithms for disorder prophylaxis in target populations.

\section{SERUM CONCENTRATIONS OF BONE TURNOVER MARKERS AND MYOKINES IN CHILDREN ON VEGETARIAN AND OMNIVOROUS DIETS}

Jadwiga Ambroszkiewiz*, Joanna Gajewska, Witold Klemarczyk, Grażyna Rowicka, Magdalena Chełchowska. Institute of Mother and Child, Warsaw, Poland

10.1136/archdischild-2021-europaediatrics.62

Vegetarian diets contain many beneficial properties but also carry a risk of inadequate intakes of several nutrients important for muscle and bone health. The links between muscle and bone have been recently intensively examined. Myokines, including myostatin and irisin are cytokines synthesized and released by muscle tissue. It is known that myokines affect bone metabolism, however, the mechanisms of these interactions are not fully understood. The aim of the study was to assess serum concentrations of bone turnover markers and myokines in prepubertal children on vegetarian and omnivorous diets.

The study included 30 healthy children (aged 5-9 years) on a lacto-ovo-vegetarian diet and 30 children on an omnivorous 
diet, who were under medical and dietary control at the Institute of Mother and Child in Warsaw (Poland). Anthropometric measurement, body composition (fat mass, lean mass, bone mineral content) and dietary constituents were assessed in all studied children. Body composition was measured by dualenergy X-ray absorptiometry. Dietary assessment was performed using a nutritional software program Dieta5. Concentrations of bone metabolism markers (osteocalcin - OC, Cterminal telopeptide of collagen type I - CTX) and myokines (myostatin and irisin) were determined in serum samples by immunoenzymatic assay. Statistical analyses were done using Statistica software. This study was approved by the Ethics Committee at the Institute of Mother and Child.

There were no significant differences in the anthropometric parameters: weight, height and body mass index in both studied groups. Compared with omnivores, vegetarians had similar bone mineral contents but lower $(\mathrm{p}<0.05)$ lean mass and percentage of fat mass. Average daily dietary energy intake was similar in both studied groups, however, vegetarians had a lower intake of protein and higher intake of carbohydrates compared with omnivores (both $\mathrm{p}<0.05$ ). The serum concentration of OC was significantly lower in children on a vegetarian diet compared with omnivores $(66.9 \pm 13.7$ vs. $85.2 \pm 20.5 \mathrm{ng} / \mathrm{mL}, \mathrm{p}<0.01)$. Additionally, levels of CTX were higher in vegetarians than in omnivores $(1.970 \pm 0.341$ vs. $1.597 \pm 0.351 \mathrm{ng} / \mathrm{mL}, \mathrm{p}<0.01)$. Serum myokines concentrations did not significantly differ in both studied groups of children, but we observed slightly higher (by about 15-20\%) levels of myostatin and irisin in vegetarians.

Our results show that a well-planned vegetarian diet with proper dairy and egg intake does not lead to changes in bone mass in prepubertal children.

However, children following a vegetarian diet had a higher rate of bone turnover and subtle changes in serum myokine levels.

\section{HEMATURIA AS AN EARLY SIGN OF MULTISYSTEM INFLAMMATORY SYNDROME IN CHILDREN (MIS-C): A CASE REPORT OF A BOY WITH MULTIPLE COMORBIDITIES}

Ana Generalić*, Maša Davidović, Ivanka Kos, Kristina Vrljičak, Lovro Lamot. University Hospital Centre Zagreb

\subsection{6/archdischild-2021-europaediatrics.63}

While clinical course of SARS-CoV-2 infection seems to be milder or asymptomatic within pediatric population, growing attention has been laid to the rare complication elicited by virus, multisystem inflammatory syndrome in children temporarily associated with COVID-19 (MIS-C). Published definition and criteria of MIS-C include persistent fever, multisystem involvement and elevated markers of inflammation, without obvious microbial inflammation or other plausible diagnosis. The aim of this case report is to emphasize diversity of symptoms of MIS-C.

We present a case of a ten-year-old boy with multiple comorbidities who developed MIS-C after asymptomatic SARS-CoV-2 infection. To our knowledge this is the first reported case of transient gross hematuria as an early sign of MIS-C. Beside hematuria, patient also presented persistent fever and elevated markers of inflammation, with no other sign of renal affection.
Moreover, he had discrete erythematous maculopapular rash on the right lower leg. Within the next two days, his condition continued to worsen despite the broad-spectrum antibiotic therapy. He started to vomit and developed abdominal pain, conjunctivitis, arrhythmia and mild left ventricular systolic dysfunction with hypotension and pleural effusion. High level of clinical suspicion for MIS-C was supported by laboratory findings (elevated ESR, CRP, proBNP, D-dimers and fibrinogen) along with positive $\operatorname{IgG}$

SARS-CoV-2 antibodies and negative microbiological cultures. The patient was given intravenous immunoglobulin (IVIG) at a dose of $2 \mathrm{~g} / \mathrm{kg}$ and began to show instantaneous clinical improvement, including downtrend of fever and inflammatory markers.

Despite the growing reports of the MIS-C in the literature, there is still paucity of studies describing the various clinical manifestation and laboratory finding in this serious condition which can be easily mistaken for many others inflammatory diseases. Therefore, pediatric professionals must be aware of (many) unusual presentations of COVID-19 associated disease in order to early recognize and treat such challenging patients.

\section{MULTISYSTEM INFLAMMATORY SYNDROME IN CHILDREN, MANAGEMENT IN A PERIPHERAL SETTING}

E Kelly*, M Jawad, M Azam, A Kalim. Wexford General Hospital

\subsection{6/archdischild-2021-europaediatrics.64}

Introduction In the context of the Covid 19 Pandemic Multisystem Inflammatory Syndrome in Children (MIS-C) is an important new differential requiring consideration, this report describes 2 cases seen in a secondary paediatric centre setting. Case Description Case 1: A 3 year-old boy presented with a four-day history of fever, malaise and decreased oral intake. The fever was persistent, it peaked at $40.8 \mathrm{C}$.

Over 4 days he had had 2 vomits but no other symptoms. He had no significant medical history and no known history of Covid 19 disease. Physical examination revealed no source of fever.

Case 2: A 14-month-old girl presented with a 1 day history of fever, malaise and decreased oral intake. She had no significant medical history and no known history of Covid 19 disease. No source of fever was evident on exam.

Initial investigations included chest $\mathrm{x}$-ray, urine culture, blood culture and throat/nasal swab for PCR for SARS CoV-2, all were negative.

Their markers of inflammation were elevated and continued to rise with persistent fever, despite treatment with IV ceftriaxone.

With no clear source of fever and no improvement, in consultation with the Infectious Diseases team in our tertiary referral hospital, we began treatment with intravenous immunoglobulin (IVIG), IV methylprednisolone and high dose aspirin for suspected MIS-C in Case 1, Case 2 was transferred to the tertiary centre for the same treatment at the ward level. With the IVIG infusion, all fever and symptoms resolved.

An Echocardiogram was organised for each in the acute setting and repeated at 6 weeks, they demonstrated normal cardiac systolic and diastolic function with no evidence of significant coronary dilatation. 